\title{
Angewandte
}

Supporting Information

(C) Wiley-VCH 2013

69451 Weinheim, Germany

\section{Optically Transparent Water Oxidation Catalysts Based on Copper} Nanowires**

Zuofeng Chen, Aaron R. Rathmell, Shengrong Ye, Adria R. Wilson, and Benjamin J. Wiley*

anie_201306585_sm_miscellaneous_information.pdf 


\section{Experimental section}

Materials: Sodium hydroxide was purchased from Fisher Scientific (S318-10). Copper(II) nitrate pentahemihydrate (12837), nickel(II) chloride hexahydrate (223387), nickel(II) nitrate hexahydrate (203874), cobalt(II) perchlorate hexahydrate (401404), ethylenediamine (EDA, E1521), 35 wt\% hydrazine in water (309400), $N$, N-diethylhydroxylamine (DEHA, 471593), polyvinylpyrrolidone MW = 10,000 (PVP10), ethyl acetate (270989), pentyl acetate (109584), and toluene (244511) were purchased from Sigma-Aldrich. White glass microscope slides $(7.5 \mathrm{~cm} \times 2.5 \times 0.1 \mathrm{~cm})$, polyethylene terephthalate (PET), and indium tin oxide (ITO) on glass or PET were purchased from McMaster-Carr. Nitrocellulose (712) was purchased from Scientific Polymer, and acetone, ethanol and isopropanol were purchased from VWR. Caution should be used when dealing with these chemicals as some are corrosive, flammable, or dangerous if ingested or put in contact with skin.

Pre-Reaction: Flasks and stir bars were cleaned with concentrated nitric acid, thoroughly rinsed with deionized water, and dried in an $80{ }^{\circ} \mathrm{C}$ oven before use. Once dry, the flasks were allowed to cool to room temperature before any reactants were added.

Reaction: $\mathrm{Cu}$ NWs were synthesized by adding $\mathrm{NaOH}(20 \mathrm{~mL}, 15 \mathrm{M}), \mathrm{Cu}\left(\mathrm{NO}_{3}\right)_{2}(1 \mathrm{~mL}, 0.1 \mathrm{M})$, EDA $(0.15 \mathrm{~mL})$, and hydrazine $(0.025 \mathrm{~mL}, 35 \mathrm{wt} \%)$ to a $50 \mathrm{~mL}$ round bottom flask. This mixture was swirled by hand for 5 seconds after each addition to mix the reactants. The solution was then heated at 80 ${ }^{\circ} \mathrm{C}$ and stirred at $200 \mathrm{rpm}$ for approximately 3 minutes. After this heating step, the solution was poured into a $50 \mathrm{ml}$ centrifuge tube, and a solution of PVP in water ( $5 \mathrm{ml}, 0.4 \mathrm{wt} \%$ ) was gently added to the top of the reaction solution. The centrifuge tube containing the reaction solution was placed in an ice bath for 1 hour. The Cu NWs floated to the top of the reaction solution and were scooped into an aqueous wash solution containing hydrazine (3 wt\%) and PVP (1 wt\%). The wash solution containing the Cu NWs was centrifuged at 2,000 rpm for 5 minutes, and the supernate was decanted from the Cu NWs. The NWs were then dispersed in new wash solution (containing $3 \mathrm{wt} \%$ hydrazine and $1 \mathrm{wt} \%$ PVP) by vortexing for 30 seconds, and then centrifuged and decanted for 3 more cycles. The Cu NWs were stored in the hydrazine (3 wt\%), PVP (1 wt\%) solution at room temperature to prevent oxidation. 
Preparation of Cu NW Transparent Electrodes: Four $20 \mathrm{~mL}$ scale reactions were combined into one centrifuge tube after the initial washing steps. This suspension was centrifuged at 2,000 rpm for 5 minutes. The $\mathrm{Cu}$ NWs were washed 3 times using a solution of hydrazine (3 wt\%) containing no PVP to ensure that most of the PVP was removed. After the PVP was removed, the Cu NWs were washed with ethanol to remove the majority of the water and then washed once more with an ink formulation described below.

The ink formulation was made by dissolving nitrocellulose (0.06 g) in acetone (2.94 $\mathrm{g})$ and then adding ethanol (3 g), ethyl acetate (0.5 g), pentyl acetate (1 g), isopropanol (1 g), and toluene (1.7 g). After the $\mathrm{Cu}$ NWs were washed with the ink formulation, another portion of ink $(0.3 \mathrm{~mL})$ was added to the $\mathrm{Cu}$ NW precipitate, and this suspension was vortexed. The final concentration of Cu NWs in the ink formulation was approximately $8 \mathrm{mg} \mathrm{mL}^{-1}$. If significant amounts of aggregates were observed by visual inspection, the ink was briefly sonicated (up to 5 seconds) and centrifuged at a low speed ( $500 \mathrm{rpm}$ ) so that a well dispersed Cu NW ink could be pipetted off from the precipitated CuNW aggregates.

To prepare transparent CuNW electrodes, glass microscope slides were placed onto a clipboard to hold them down while the $\mathrm{Cu}$ NW ink $(30 \mu \mathrm{L})$ was pipetted in a line at the top of the glass. A Meyer rod (Gardco \#13, $33.3 \mu \mathrm{m}$ wet film thickness) was then quickly $(<1$ second) pulled down over the Cu NW ink by hand, spreading it across the glass to form a thin, uniform film. The density of nanowires on the surface of the substrate could be adjusted by varying the concentration of the Cu NWs in the ink.

The CuNW films were not conductive before being cleaned in a plasma cleaner (Harrick Plasma PDC- 001) for 15 minutes in an atmosphere of 95\% nitrogen and 5\% hydrogen at a pressure of 600-700 mTorr to remove the nitrocellulose. To further clean the Cu NW electrodes, the NW-coated glass samples were heated to $175^{\circ} \mathrm{C}$ in a tube furnace for 30 minutes under a constant flow of hydrogen $\left(600 \mathrm{~mL} \mathrm{~min}^{-1}\right)$ to anneal the wires together and decrease the sheet resistance to its final value.

Preparation of $\mathrm{Cu}_{c}-\mathrm{Ni}_{s} \mathrm{NW}$ Transparent Electrodes: CuNW films were coated with a layer of Ni by electroplating $\mathrm{Ni}(\mathrm{II})(0.5 \mathrm{mM})$ on $\mathrm{Cu} \mathrm{NW}$ networks in $0.2 \mathrm{M}$ deaerated borate buffer (pH 9.2) with the model CHI601D electrochemical workstation (CH Instruments, Austin, TX) at room temperature. Electroplating was conducted by holding the potential at $-0.75 \mathrm{~V}$ vs. NHE for different times to obtain 
different degrees of $\mathrm{Ni}$ deposition on the NW network. Prior to use as a catalyst, the as-prepared $\mathrm{Cu}_{c}-\mathrm{Ni}_{\mathrm{s}}$ $\mathrm{NW}$ networks could be stored in air with no appreciable degradation of the samples. The $\mathrm{Cu}_{\mathrm{c}}-\mathrm{Ni}_{\mathrm{s}} \mathrm{NW}$ transparent films on PET (cut into a $7.5 \mathrm{~cm} \times 2.5 \mathrm{~cm}$ strip) were prepared by the same method. The $\mathrm{Cu}_{\mathrm{c}^{-}}$ $\mathrm{Co}_{\mathrm{s}} \mathrm{NW}$ transparent films were prepared by holding the potential at $-0.75 \mathrm{~V}$ vs. NHE for 20 min in $0.2 \mathrm{M}$ deaerated phosphate buffer ( $\mathrm{pH} 7.0)$, with $0.5 \mathrm{mM} \mathrm{Co}\left(\mathrm{ClO}_{4}\right)_{2}$ added to solution.

The $\mathrm{NiO}_{\mathrm{x}} \mathrm{H}_{\mathrm{y}}$ cluster film on an ITO-coated glass substrate was prepared by following a previously published procedure, but without stirring the solution. ${ }^{[1]}$ Briefly, Ni(II) ions $(0.5 \mathrm{mM})$ were anodically deposited onto an ITO film in $0.2 \mathrm{M}$ borate buffer ( $\mathrm{pH} 9.2$ ) at $1.30 \mathrm{~V}$ vs. NHE for $1 \mathrm{~h}$, at which point a catalytic current density of $0.8 \mathrm{~mA} / \mathrm{cm}^{2}$ was reached. This geometrical current density is equivalent to that of a $\mathrm{Cu}_{\mathrm{c}}-\mathrm{Ni}_{\mathrm{s}} \mathrm{NW}$ network made with a Cu NW density of $65 \mathrm{mg} \mathrm{m}^{-2}$.

A piece of polycrystalline $\mathrm{Cu}$ or $\mathrm{Ni}$ foil was used to evaluate the active surface areas of $\mathrm{Cu} \mathrm{NW}$ and $\mathrm{Cu}_{\mathrm{c}}-\mathrm{Ni}_{\mathrm{s}} \mathrm{NW}$ networks by comparing the double layer charging current. The double layer charging current was calculated by taking the difference between the cathodic and anodic current in the approximate center of the double layer charging region $(-0.40 \mathrm{~V}$ vs. NHE for $\mathrm{Cu},-0.35 \mathrm{~V}$ vs. NHE for $\mathrm{Ni})$ on the CV scan (see Figure S1). The ratio of the double layer charging current between the NW film and the foil was taken to be approximately equivalent to the ratio of their active surface areas. Prior to measurement, the $\mathrm{Cu}$ and $\mathrm{Ni}$ foil were cleaned by ultrasonication in acetone for $5 \mathrm{~min}$, and then in $0.1 \mathrm{M} \mathrm{H}_{2} \mathrm{SO}_{4}$ for 2 min.

Characterization: The transmittance and sheet resistance of the NW electrode were measured using a UV/VIS spectrometer (Cary 6000i) and a four-point probe (Signatone SP4-50045TBS), respectively. Every reported value of the sheet resistance is the average of 5 measurements. In the case of the $\mathrm{Cu}_{\mathrm{c}}-\mathrm{Ni}_{\mathrm{s}}$ NW film after water oxidation, the surface of the NWs is covered by a $\mathrm{Ni}(\mathrm{OH})_{2}$ and/or $\mathrm{NiOOH}$ layer, which provides poor electrical contact with the four-point probe. In this case, electrical contact was made to the film with silver paste, and the resistance of a square was measured to obtain the sheet resistance.

The films were imaged with a dark field optical microscope (Olympus BX51 fitted with a digital SC30 CMOS camera) to show the density, distribution, and color of nanowires in the film. 
To prepare the samples for SEM (FEI XL30 SEM-FEG), a small chip of NW film on glass (5 mm X $5 \mathrm{~mm}$ ) was placed on a piece of double sided conductive carbon tape on an SEM sample platform. The sample surface was then partially covered with carbon tape to electrically connect it to the platform, leaving a small spot uncovered for SEM imaging.

To prepare the samples for TEM (FEI Tecnai G ${ }^{2}$ Twin), the Cu NWs and $\mathrm{Cu}_{\mathrm{c}}-\mathrm{Ni}_{\mathrm{s}} \mathrm{NWs}$ were carefully removed from the glass slide by a razer blade and were dispersed in methanol by sonicating for 2 seconds. Copper grids coated with a thin film of carbon coated 400 mesh $\mathrm{Cu}$ grid (SPI, \#3540C-FA) were used to support the nanowires. Each sample, once deposited on a grid, was allowed to completely dry under a flow of nitrogen gas.

X-ray photoelectron spectroscopy (XPS) for elemental analysis was conducted on a Kratos Axis Ultra DLD X-ray Photoelectron Spectrometer. The systematic error/drift of the instrument was corrected by comparasion of the experimental C 1s signal to C 1s standard at $284.6 \mathrm{eV}$.

Concentration of $\mathrm{Cu} N W s$ and $\mathrm{Cu}_{c}-\mathrm{Ni}_{s} \mathrm{NWs}$ : To measure the concentration of the well-dispersed $\mathrm{Cu}$ NW suspension, a set volume of the suspension was dissolved in concentrated nitric acid (1 ml). The dissolved copper was then diluted to a set volume, and its concentration was analyzed using an atomic absorption spectrometer (AAS, Perkin Elmer 3100).

To measure the mass percentage of $\mathrm{Ni}$ in $\mathrm{Cu}_{\mathrm{c}}-\mathrm{Ni}_{\mathrm{s}} \mathrm{NW}$ networks, a piece of an as-prepared $\mathrm{Cu}_{\mathrm{c}}-\mathrm{Ni}_{\mathrm{s}}$ NW film was dissolved in concentrated nitric acid $(1 \mathrm{ml})$. Both $\mathrm{Cu}$ and $\mathrm{Ni}$ concentrations were then measured via AAS in the same way as above.

Testing the Flexibility of Nanowire Films on PET: To test the flexibility of the $\mathrm{Cu}_{\mathrm{c}}-\mathrm{Ni}_{\mathrm{s}} \mathrm{NW}$ films, we attached one end of the film to a tabletop and the other end to a ruler which was on top of a spring. The set-up was designed so that the initial radius of curvature was $10 \mathrm{~mm}$ (no force applied to the ruler) and after a downward force was applied to the ruler the final radius of curvature was $2.5 \mathrm{~mm}$. The ruler was then allowed to spring back to the starting position and the entire cycle was counted as 1 . The sheet resistance was measured every 200 cycles. 
Electrocatalytic Water Oxidation. Electrochemical measurements were performed with the model CHI601D electrochemical workstation (CH Instruments, Austin, TX). The three-electrode system consisted of a working electrode, a coiled platinum wire counter electrode, and a saturated calomel reference electrode (SCE, $\sim 0.244 \mathrm{~V}$ vs. NHE). To measure the overpotential at a current density of 1 $\mathrm{mA} / \mathrm{cm}^{2}$, electrolysis was performed while stirring the solution, and with compensation for the resistance of the solution. The solution resistance $(\sim 30 \Omega)$ was measured with a clean $\mathrm{Cu}$ foil working electrode using the $i \mathrm{R}$ test function prior to data collection. Unless stated otherwise, all electrochemical experiments were conducted without sitrring the solution and all potentials were reported vs. NHE without $i R$ compensation.

CV measurements for catalytic water oxidation were conducted without deaeration of the solution. Measurements of the oxygen evolved from the electrode during electrolysis were conducted with the solution and its headspace purged by an Ar stream for about 15 min prior to measurement. The electrolysis was conducted under an atmosphere of Ar. The oxygen evolved was measured with an oxygen probe (YSI ProODO).

All electrochemical experiments were performed at room temperature $\left(20 \pm 2{ }^{\circ} \mathrm{C}\right)$.

\section{Reference:}

[1] M. Dincă, Y. Surendranath, D. G. Nocera, P. Natl. Acad. Sci. USA 2010, 107, 10337-10341. 

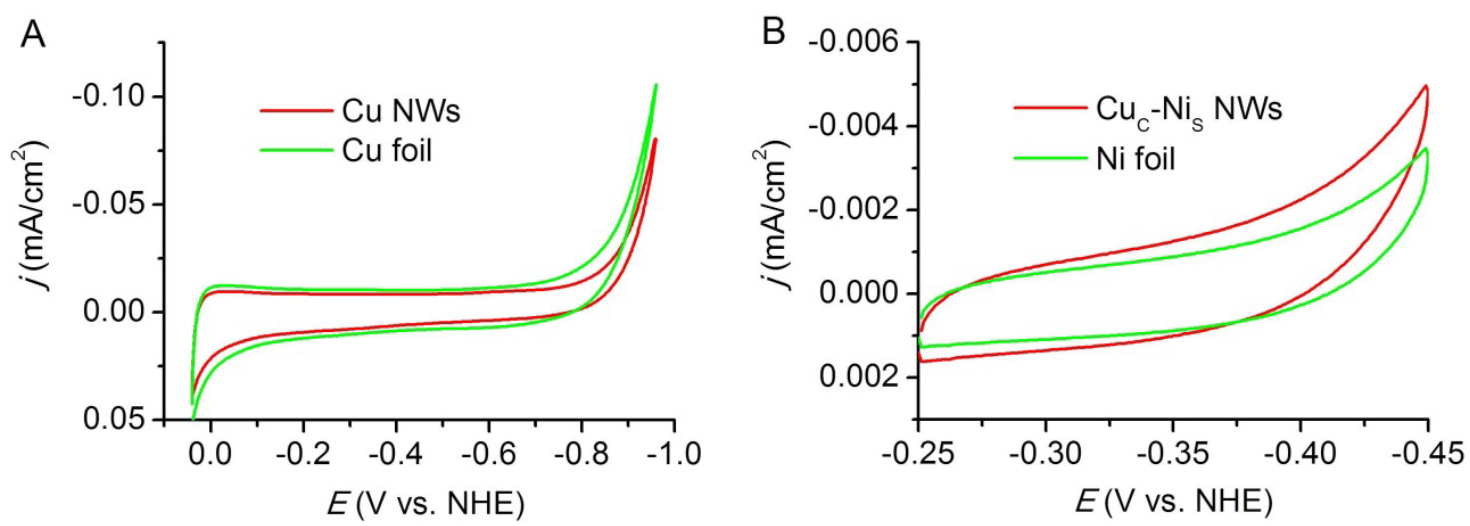

Figure S1. (A) Cyclic voltammograms of a Cu NW network and a polycrystalline copper foil of the same geometric area. Scan rate, $100 \mathrm{mV} / \mathrm{s}$. (B) Cyclic voltammograms of a $\mathrm{Cu}_{\mathrm{c}}-\mathrm{Ni}_{\mathrm{s}} \mathrm{NW}$ network and a polycrystalline nickel foil of the same geometric area. Scan rate, $10 \mathrm{mV} / \mathrm{s}$. Solution, $0.2 \mathrm{M}$ deaerated borate buffer (pH 9.2); 

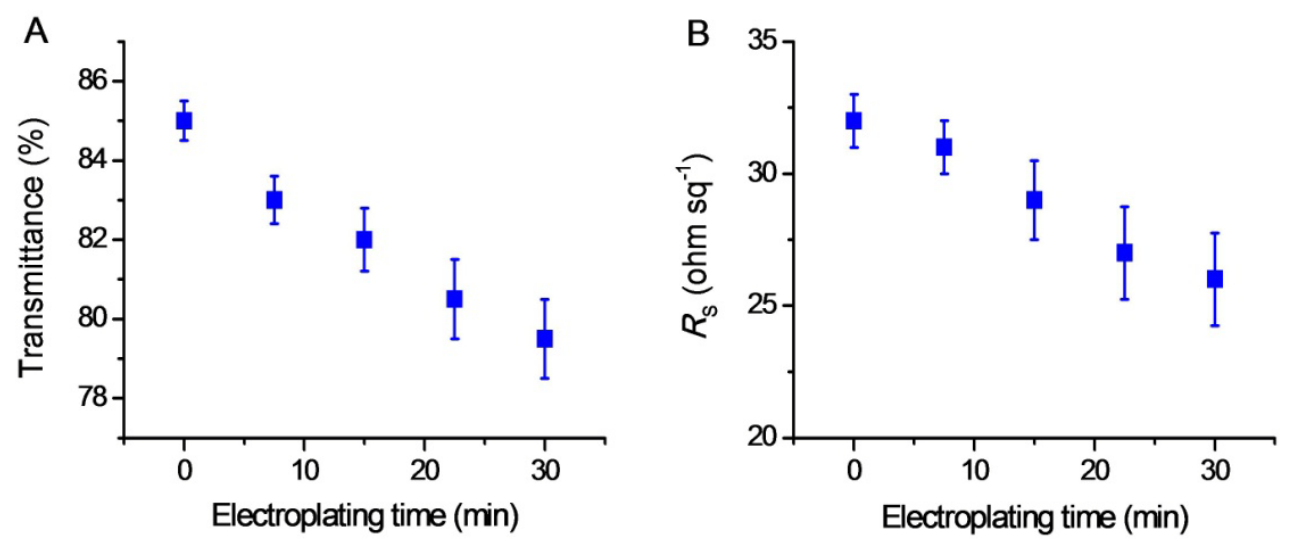

Figure S2. (A) A plot of transmittance vs. electroplating time shows the effect of increasing Ni content on the film's transmittance. (B) A plot of sheet resistance $\left(R_{\mathrm{S}}\right)$ vs. electroplating time shows the effect of increasing Ni content on the film's sheet resistance. 


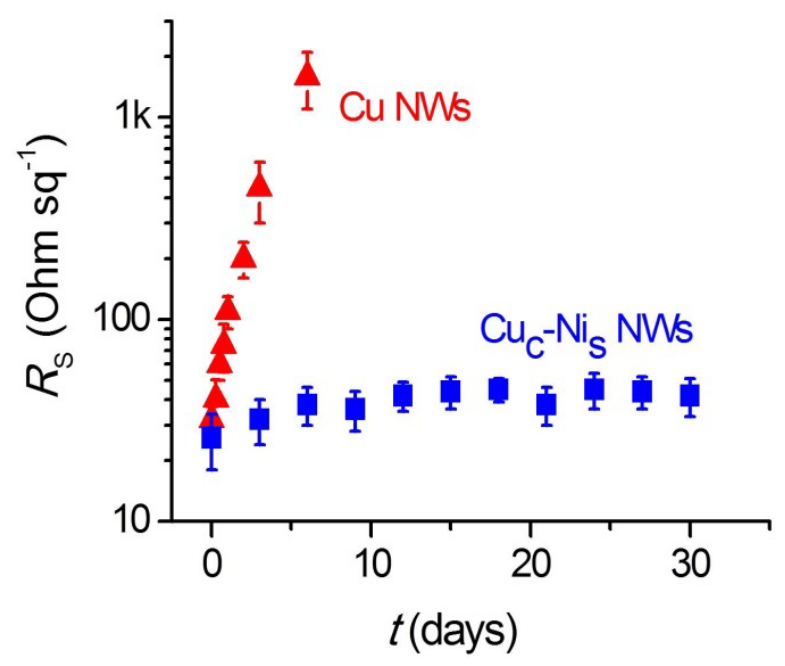

Figure S3. Plots of sheet resistance $\left(R_{\mathrm{S}}\right)$ vs. time for films of Cu NWs (32 $\Omega \mathrm{sq}^{-1}$ initially) and $\mathrm{Cu}_{\mathrm{c}}-\mathrm{Ni}_{\mathrm{s}}$ NWs (33 wt\% Ni, $26 \Omega \mathrm{sq}^{-1}$ ) stored in an oven at $85^{\circ} \mathrm{C}$. 


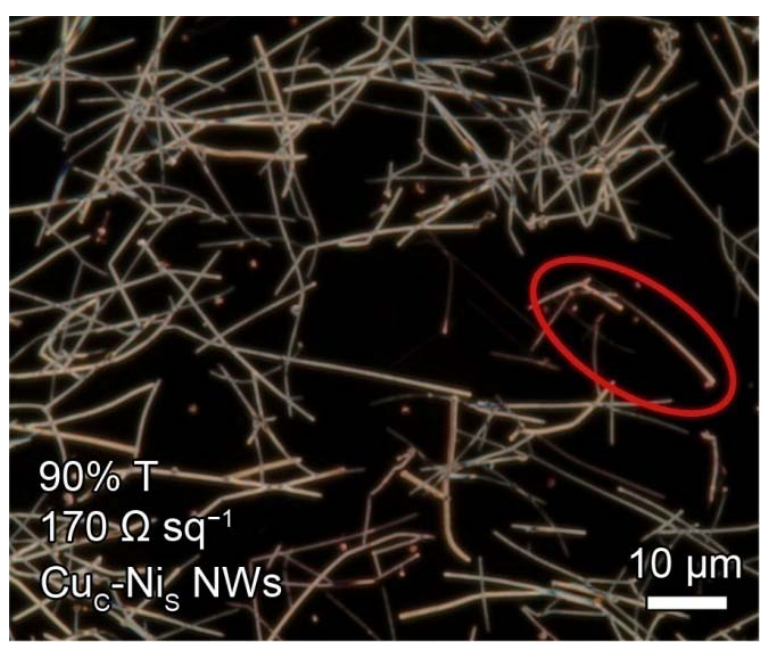

Figure S4. Dark field optical microscope image of $\mathrm{Ni}$ electroplated on a $\mathrm{Cu} \mathrm{NW}$ network of lower density (90\% transmittance, $170 \Omega \mathrm{sq}^{-1}$ ). Other conditions are identical to that in Figure 1. An example of an isolated $\mathrm{Cu} \mathrm{NW}$ with no Ni coating is circled in red. 

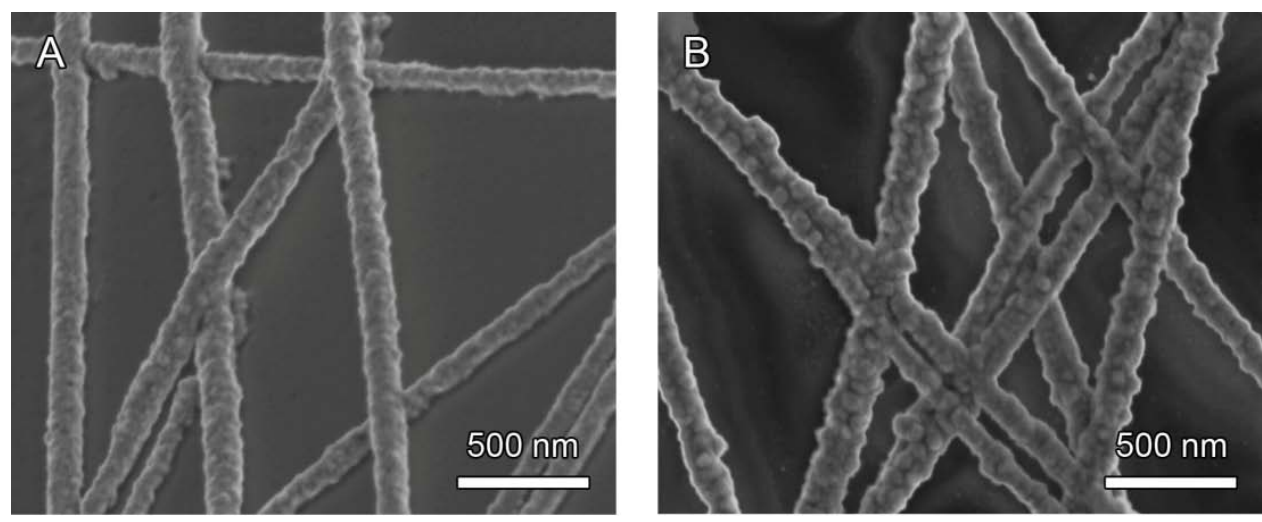

Figure S5. SEM image of $\mathrm{Cu}_{\mathrm{c}}-\mathrm{Ni}_{\mathrm{s}} \mathrm{NW}$ networks prepared by electroplating at (A) $-0.80 \mathrm{~V}$ and (B) -0.85 V for 30 min. 

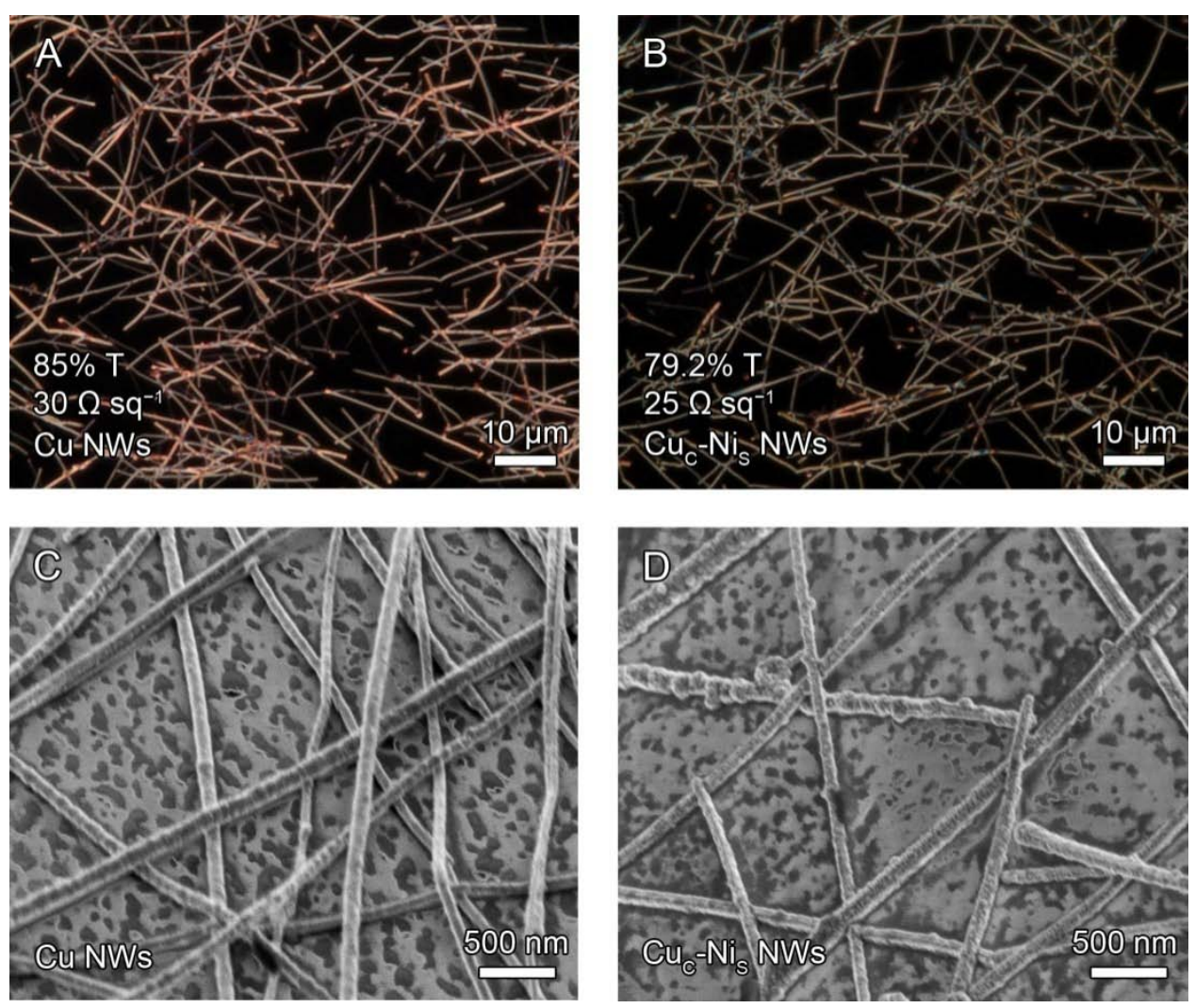

Figure S6. Dark field optical microscope (upper) and SEM (lower) images of a Cu NW network (left) and a $\mathrm{Cu}_{\mathrm{c}}-\mathrm{Ni}_{\mathrm{s}}(33 \% \mathrm{Ni}) \mathrm{NW}$ network (right) on PET. 

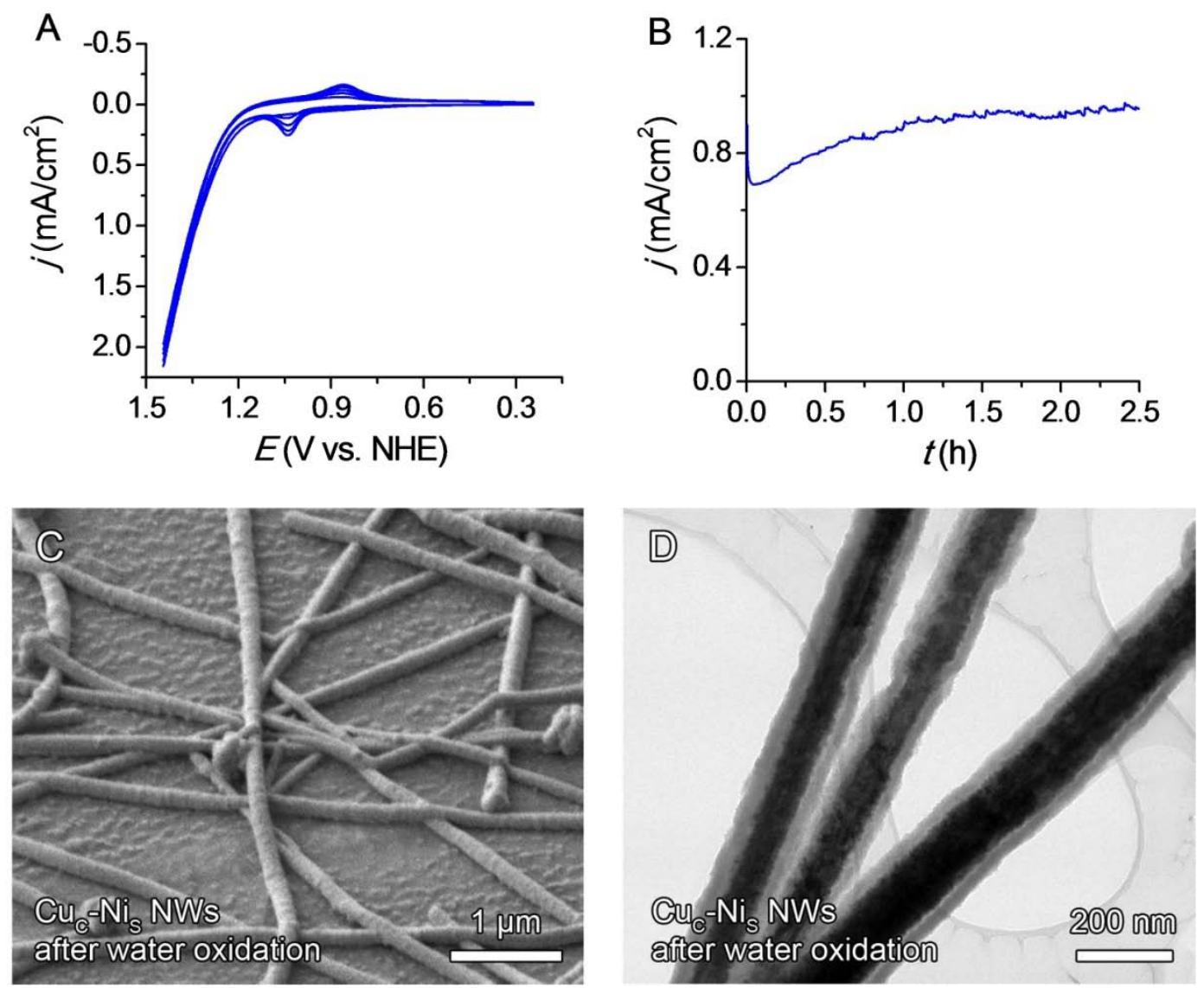

Figure S7. (A) Cyclic voltammograms of $\mathrm{Cu}$ (red) and $\mathrm{Cu}_{\mathrm{c}}-\mathrm{Ni}_{\mathrm{s}}$ (blue) $\mathrm{NW}$ networks on PET in $0.2 \mathrm{M}$ borate buffer (pH 9.2). Scan rate, $100 \mathrm{mV} / \mathrm{s}$. (B) Controlled potential electrolysis of the $\mathrm{Cu}_{\mathrm{c}}-\mathrm{Ni}_{\mathrm{s}} \mathrm{NW}$ 
network on PET at $1.30 \mathrm{~V}$ vs. NHE with $0.03 \mathrm{mM} \mathrm{Ni}\left(\mathrm{NO}_{3}\right)_{2}$ added to the solution. (C) SEM and (D) TEM images of $\mathrm{Cu}_{\mathrm{c}}-\mathrm{Ni}_{\mathrm{s}} \mathrm{NW}$ networks after electrolysis.
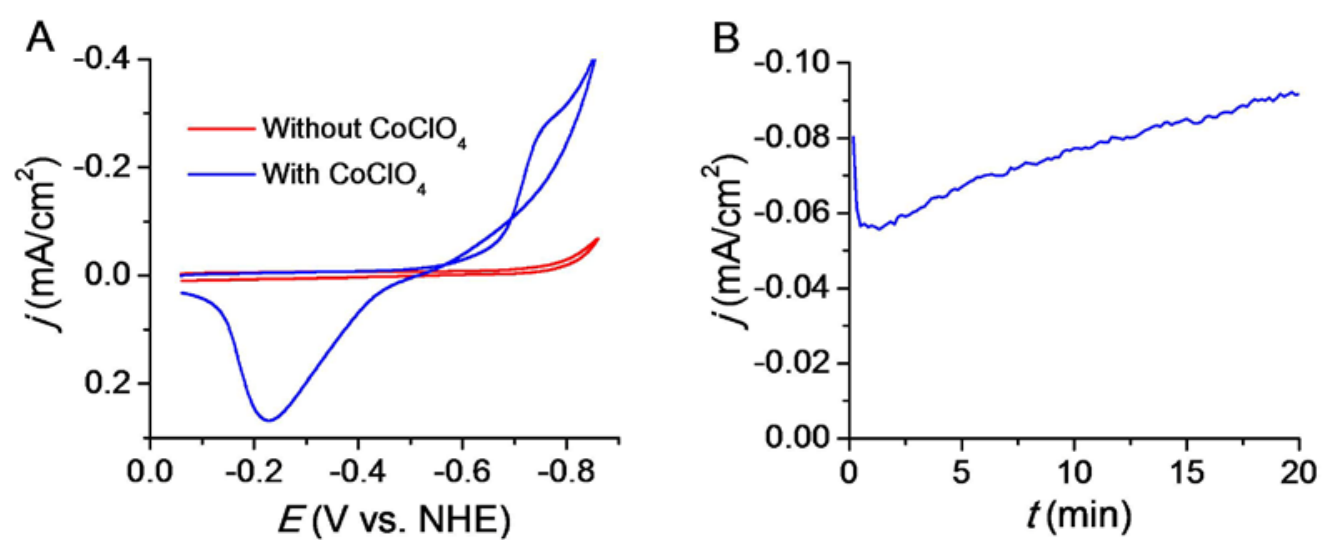

Figure S8. (A) Cyclic voltammograms of a Cu NW network in $0.2 \mathrm{M}$ phosphate buffer (pH 7.0) with and without $0.5 \mathrm{mM} \mathrm{Co}\left(\mathrm{ClO}_{4}\right)_{2}$ added to the solution. Scan rate, $100 \mathrm{mV} / \mathrm{s}$. (B) Controlled potential electroplating of Co onto $\mathrm{Cu}$ NWs at $-0.75 \mathrm{~V}$ vs. NHE with $0.5 \mathrm{mM} \mathrm{Co}\left(\mathrm{ClO}_{4}\right)_{2}$ added to the solution. 